\title{
ELABORAÇÃO DE UM PLANO DE CUIDADOS SISTEMATIZADO PARA UMA PACIENTE COM COLESTASE INTRA-HEPÁTICA GESTACIONAL
}

\section{Ana Júlia Góes Maués ${ }^{1}$; Rosália Cardoso da Silva²; Sabrina de Lucas Ramos Necy ${ }^{3}$; Zayra Elizandra Santos Sena ${ }^{4}$; Gleiciene Oliveira Borges ${ }^{5}$; Victória Lima Mendes Leite ${ }^{6}$; Rebeca Prata Meireles ${ }^{7}$; Fernanda Cristina Silva da Silva ${ }^{8 ;}$ Marcos José Risuenho Brito ${ }^{9}$; Marcelo Williams Oliveira de Souza ${ }^{10 .}$}

Acadêmica de enfermagem, Universidade do Estado do Pará (UEPA), Belém, Pará. Acadêmica de enfermagem, Universidade do Estado do Pará (UEPA), Belém, Pará. Acadêmica de enfermagem, Universidade do Estado do Pará (UEPA), Belém, Pará. Acadêmica de enfermagem, Universidade do Estado do Pará (UEPA), Belém, Pará. Acadêmica de enfermagem, Universidade do Estado do Pará (UEPA), Belém, Pará. Acadêmica de enfermagem, Universidade do Estado do Pará (UEPA), Belém, Pará. Acadêmica de enfermagem, Universidade do Estado do Pará (UEPA), Belém, Pará. Acadêmica de enfermagem, Universidade do Estado do Pará (UEPA), Belém, Pará. Enfermeiro. Universidade do Estado do Pará (UEPA), Belém, Pará. Enfermeiro. Universidade do Estado do Pará (UEPA), Belém, Pará.

\section{DOI: 10.47094/IICNNESP.2021/155}

PALAVRAS-CHAVE: Cuidados de Enfermagem; Assistência Centrada no Paciente; Enfermagem Obstétrica.

ÁREA TEMÁTICA: Outros.

\section{INTRODUÇÃO}

A gestação é um período de importantes modificações hormonais que desencadeiam alterações na fisiologia de todo o organismo materno. O fígado, maior glândula e o segundo maior órgão do corpo humano, cursa com substancial adaptação ao estado gestacional, podendo também interferir na evolução da gravidez (POLLO FLORES, 2015). Dentre as doenças hepáticas específicas da gravidez, pode-se citar a Colestase Intra-Hepática Gestacional (LEE, 2018). Caracteriza-se, principalmente, por prurido, marcadamente nas palmas das mãos e pés, mas que pode acometer todo o corpo, geralmente aumentando de intensidade com a progressão da gestação e de resolução no puerpério. Pode também estar associado a outros sintomas e sinais como mal-estar, dor abdominal, urina escurecida, fezes esbranquiçadas e esteatorréia (PACHECO et al, 2019). Embora a evolução clínicolaboratorial dessa colestase seja considerada benigna para a mãe, esta afecção tem sido associada a 
desfechos obstétricos inexplicavelmente insatisfatórios, como parto prematuro, presença de mecônio, bradicardia fetal, sofrimento fetal e até óbito do concepto; devendo, portanto, ser considerada como gestação de alto risco (SOUZA et al, 2014). Diante disso, faz-se necessário à elaboração de um plano de cuidados sistematizado durante o pré-natal das pacientes que apresentam essa patologia, com o intuito de promover um cuidado mais humanizado e holístico, assim como um desfecho clínico mais favorável. Portanto, o objetivo deste trabalho é relatar a experiência de acadêmicas de enfermagem no desenvolvimento de um plano de cuidados sistematizado baseado na taxonomia presente na Nursing Diagnosis Association (NANDA), Nursing Outcomes Classification (NOC) e Nursing Interventions Classification (NIC) para uma paciente com diagnóstico de Colestase Intra-Hepática Gestacional.

\section{METODOLOGIA}

Trata-se de um estudo descritivo do tipo relato de experiência que ocorreu no decorrer das aulas práticas do componente curricular Enfermagem Obstétrica da Universidade do Estado do Pará (UEPA), em um hospital público de grande porte do município de Belém-PA, no período de 06 a 08 de novembro de 2019. Os dados coletados foram obtidos a partir da anamnese, exame físico e consulta ao prontuário de uma paciente do sexo feminino de 39 anos, secundigesta, multípara, que foi admitida no pré-natal de alto risco. A partir das informações coletadas, efetuou-se a elaboração dos diagnósticos e prescrições referentes ao plano assistencial. Durante o primeiro contato das acadêmicas com a usuária, realizou-se a anamnese, através de escuta ativa e coleta de dados e, em seguida foi realizado um exame físico criterioso. Logo, baseado nos elementos que foram coletados foi possível definir os diagnósticos de enfermagem que serviram de suporte para determinar os resultados e intervenções que seriam realizadas face às respostas obtidas.

\section{RESULTADOS E DISCUSSÕES}

Como resultados foram identificados os principais diagnósticos de enfermagem: 1- Motilidade gastrintestinal disfuncional relacionada à colestase hepática e fluxo irregular da bile evidenciada por dificuldade para defecar e fezes endurecidas; 2-Distúrbio no padrão de sono relacionado a padrão de sono não restaurador evidenciado por dificuldade no desempenho das funções diárias e Privação do sono relacionado ao prurido intenso durante a noite evidenciado por fadiga; 3- Risco de integridade da pele prejudicada relacionada ao prurido intenso durante a noite; 4- Risco de Infecção relacionada a alteração na integridade da pele; 5- Risco de queda relacionado a sonolência intensa; 6- Conforto prejudicado relacionado ao prurido intenso evidenciado por alteração no padrão do sono. Os respectivos resultados de enfermagem foram: 1- Aumento da motilidade gastrointestinal funcional; 2- Padrão de sono melhorado; 3- Integridade da pele preservada; 4- Controle de riscos: processo infeccioso; 5- Comportamento de prevenção de quedas; 6- Estado de conforto. As respectivas intervenções de enfermagem foram: 1- Realizar ausculta abdominal em busca de ruídos hidroaéreos e promover ingestão nutricional adequada; 2- Estimular o repouso sempre que possível e oferecer condições adequadas para que ele ocorra; 3-Orientar a paciente a manter as unhas curtas para evitar lacerações 
na pele, oferecer hidratante corporal, assim como orientar seu uso adequado, evitar perfumes e outros cosméticos que contenham álcool e estimular banho com água fria; 4- Examinar pele e mucosas em busca de hiperemia, calor extremo ou lacerações e aconselhar paciente e família sobre formas de evitar infecção; 5- Evitar tapetes e objetos no chão e sempre estar observando o paciente; 6- Oferecer condições adequadas para o conforto.

\section{CONCLUSÃO}

Sendo assim, a sistematização da assistência de enfermagem como ferramenta metodológica da equipe de enfermagem para direcionar e prestar cuidado a paciente diagnosticada com colestase intra-hepática gestacional é de suma importância para o desenvolvimento da gestação e do feto,visto que o desenvolvimento de um plano assistencial sistematizado de enfermagem, elaborado a partir de buscas nas bases cientificas visando atender o binômio mãe-feto de forma holística é fundamental, dado que após a aplicação do mesmo, foi possível observar uma melhora significativa na qualidade de vida da paciente durante a sua internação, principalmente relacionada a melhora no seu padrão de sono, uma melhora da motilidade intestinal, promoção da integridade da pele, prevenção de infecções. Isto evidencia que uma assistência realizada de forma sistematizada é fundamental não só para a identificação das demandas específicas do usuário, mas para que seja possível obter um cuidado mais integral e humanizado para o mesmo.

\section{PRINCIPAIS REFERÊNCIAS}

POLLO-FLORES, P. et al. Alterações hepáticas da gravidez. Femina, São Paulo, v. 43, n. 5, p.225234, out. 2015. Disponível em: https://pesquisa.bvsalud.org/portal/resource/pt/lil-771218?lang=pt. Acesso em: 18 mai. 2021.

LEE, R. H; TRAN, T. Approach to liver disease occurring during pregnancy. UpToDate, p.111, jan. 2018. Disponível em: https://www.uptodate.com/contents/approach-to-liver-diseaseoccurring-during-pregnancy?source=search_result\&search=acute $\% 20$ fatty $\% 201$ iver $\% 20$ of $\% 20$ pregnancy\&selectedTitle=4 40. Acesso em: 18 mai. 2021.

PACHECO A.J.C et al. Colestase Intra-Hepática na gravidez: Relato de Caso. Arquivos de saúde biologia e sociedade. Bahia, v.1, n.1, p.1-10, 2019. Disponível em:http://revistaadmmade.estacio.br/ index.php/asbsestaciojuazeiro/article/view/7561/47966342. Acesso em: 17 mai. 2021.

SOUZA, Eduardo de et al. Colestase intra-hepática da gravidez: evidências científicas para escolha do tratamento. FEMINA. v.42, n.1, p.39-42, 2014. Disponível em: http://files.bvs.br/ upload/S/0100-7254/2014/v42n1/a4812.pdf. Acesso em: 18 mai. 2021. 